\title{
Presencia de metales pesados y calidad microbiológica de concentrados para la alimentación porcina en cuatro regiones colombianas
}

\author{
Presence of heaVy Metals AND Microbiological quality of CONCENTRATES \\ FOR PIG FEEDING IN FOUR COLOMBIAN REGIONS

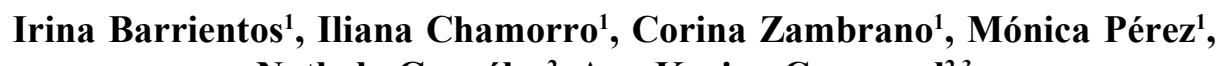 \\ Nathaly González ${ }^{2}$, Ana Karina Carrascal ${ }^{2,3}$
}

\section{Resumen}

\begin{abstract}
El objetivo del trabajo fue evaluar la presencia de metales pesados (arsénico, cadmio, mercurio y plomo), Salmonella y E. coli, así como el recuento de mesófilos, hongos y levaduras y el recuento de Clostridium sulfito reductor en concentrados destinados para la alimentación de cerdos de cuatro regiones de Colombia. Se recolectaron 38 muestras de concentrados de 19 granjas para el análisis de metales pesados. Las muestras se procesaron por triplicado y se analizaron mediante espectrometría de absorción atómica acoplada a plasma. Para el análisis microbiológico se procesaron 391 muestras de 22 granjas. Para la detección de Salmonella se usó el equipo MDS-3M y su presencia se confirmó usando Maldi-tof, en tanto que para el recuento de E. coli se usó agar cromógeno (Petrifilm); para los aerobios mesófilos se empleó el agar plate count, y para hongos y levaduras el agar rosa de bengala. Los resultados indicaron que solo dos muestras de una misma granja presentaron valores elevados de arsénico ( 2 ppm) mientras que los demás metales estuvieron dentro de los límites esperados. Los recuentos de mesófilos, hongos y levaduras y Clostridium estuvieron dentro de la reglamentación colombiana, habiendo solo una muestra positiva para Salmonella $(0.25 \%)$, en tanto que el $1 \%$ de las muestras presentó E. coli. Los resultados reflejan que la calidad de los concentrados que se emplean en la alimentación porcina no son una fuente importante de patógenos o de metales pesados.
\end{abstract}

Palabras clave: alimentación; porcinos; metales pesados; análisis microbiológico; concentrados

\footnotetext{
${ }^{1}$ Centro de Investigación y Transferencia de Tecnología del Sector Porcícola - CENIPORCINO, Asociación Porkcolombia - Fondo Nacional de la Porcicultura, Colombia

${ }^{2}$ Laboratorio de Microbiología de Alimentos, Grupo de Biotecnología Ambiental e Industrial, Departamento de Microbiología, Facultad de Ciencias, Pontificia Universidad Javeriana, Bogotá, Colombia

${ }^{3}$ E-mail: acarrasc@javeriana.edu.co
}

Recibido: 17 de noviembre de 2017

Aceptado para publicación: 20 de abril de 2018 
The objective of this study was to evaluate the presence of heavy metals (arsenic, cadmium, mercury, and lead), Salmonella and E. coli, as well as mesophiles, fungi and yeast counts and Clostridium sulfite reductor count in concentrates for pigs in four regions of Colombia. Feed samples ( $\mathrm{n}=38$ ) were collected from 19 farms for the analysis of heavy metals. Samples were processed in triplicate and analyzed by plasma-coupled atomic absorption spectrometry. For the microbiological analysis, 391 samples from 22 farms were processed. For the detection of Salmonella, the MDS-3M equipment was used, and its presence was confirmed using Maldi-tof, while for the counting of $E$. coli cromocult agar was used. For mesophilic aerobes, the plate count agar was used and for the fungi and yeasts rose bengal agar. The results indicated that only two samples from the same farm had high arsenic values (2 ppm) while the other metals were within the expected limits. The counts of mesophiles, fungi and yeasts and Clostridium were within the Colombian regulations, with only one positive sample for Salmonella $(0.25 \%)$, while $1 \%$ of the samples presented E. coli. The results reflect that the quality of concentrates used in pig feeding is not an important source of pathogens or heavy metals.

Key words: feeding; pigs; heavy metals; microbiological analysis; concentrates

\section{INTRODUCCIÓN}

Los molinos productores de concentrados animales son importantes proveedores de las granjas porcinas en las explotaciones intensivas, donde los costos asociados a la alimentación rondan entre el 40 y $60 \%$ (den Hartog, 2003). La calidad nutricional ha sido durante muchos años uno de los aspectos más importantes a considerar; sin embargo, la presencia de dioxinas en carnes de cerdo asociadas al consumo de concentrados animales (Hoogenboom et al., 2004; Bernard et al., 2002) ha determinado que la alimentación animal puede ser considerado como un peligro potencial en la salud pública (den Hartog, 2003).

Las sustancias tóxicas tales como dioxinas, micotoxinas, metales pesados, pesticidas, residuos de medicamentos e hidrocarburos aromáticos policíclicos pueden llegar a estar presentes en los ingredientes para la alimentación animal (Kant y Meijeir, 2007). Dentro de los peligros biológicos se incluye la presencia de Salmonella (Baer et al.,
2013), bacteria que puede localizarse en los ganglios linfáticos de cerdos (Boughton et al., 2004) y ser excretada en las heces durante toda la vida productiva del cerdo; además, puede contaminar la canal durante el sacrificio y causar salmonelosis en humanos por el consumo de carne contaminada (Binter et al., 2011). Así mismo, diversos estudios han demostrado que las cepas de Salmonella aisladas de concentrados en las etapas de finalización han sido, además, aisladas de las carcasas durante el proceso de beneficio y desposte (Shirota et al., 2001; Österberg et al., 2006; Molla et al., 2010).

La producción de cerdos ha crecido gradualmente en Colombia, alcanzando altos niveles de tecnificación, especialmente en la cadena primaria. En este sentido, las granjas porcinas emplean concentrados elaborados por empresas de alto reconocimiento en el país. Adicionalmente, la calidad de los concentrados en el país es controlada por el Instituto Colombiano Agropecuario (ICA), mediante la disposición DIP-30-100.003, donde se incluyen dentro de los análisis microbiológicos, el recuento de bacterias 
Cuadro 1. Análisis microbiológicos para alimentos concentrados

\begin{tabular}{|c|c|c|}
\hline Análisis & Técnica empleada & $\begin{array}{l}\text { Referencia } \\
\text { bibliográfica }\end{array}$ \\
\hline Bacterias aerobias mesófilas & Recuento en placa (agar plate count) & APHA (2015) \\
\hline Hongos y levaduras & $\begin{array}{l}\text { Recuento en placa (agar rosa de } \\
\text { bengala) }\end{array}$ & APHA (2015) \\
\hline Escherichia coli & Recuento en Petrifilm & APHA (2015) \\
\hline Clostridium sulfito reductor & Recuento en placa (agar TSN) & APHA (2015) \\
\hline Salmonella spp & Sistema Molecular MDS de 3M & Bird et al. (2016) \\
\hline
\end{tabular}

mesófilas, recuento de hongos y levaduras, recuentos de Escherichia coli, recuento de Clostridium sulfito reductory Salmonella spp. No obstante, información sobre la calidad de los concentrados empleados en alimentación de cerdo en Colombia es escasa y se concentra en la evaluación de la calidad nutricional (Borbolla et al., 2002). Por esta razón, el objetivo de este trabajo fue evaluar la presencia de metales pesados y la calidad microbiológica en concentrados para cerdos empleados en las diferentes etapas de producción en granjas tecnificadas del país.

\section{Materiales y Métodos}

Se seleccionaron 22 granjas de productores ubicadas en los departamentos con la mayor población porcina de Colombia (ICA, 2016). Las granjas fueron de Antioquia (13), Valle del Cauca (5), Meta (1) y Cundinamarca (3). Las granjas incluidas participaron de manera voluntaria. El muestreo se realizó de manera estratificada y por fijación proporcional.

\section{Análisis Microbiológicos}

El tamaño de muestra fue calculado mediante la estimación puntual de la prevalencia en el programa estadístico Nuevo Ta- maño de Muestra 1.1. Teniendo en cuenta que el patógeno de interés en este producto es Salmonella y al no tener información sobre su prevalencia en Colombia, se estableció una prevalencia esperada del 50\%; asimismo, se tomó como base de la población el inventario de cerdos en los predios de 2013 (información suministrada por Porkcolombia, Fondo Nacional de la Porcicultura), el cual fue de 6140762 animales. El tamaño de muestra resultante fue de 385 muestras como mínimo. Se asumió un error tipo I del 5\%.

El muestreo se realizó una vez por granja, tomándose tres muestras de concentrado de cada etapa productiva (reemplazo, gestación, lactancia, precebos, levante, ceba y reproducción). Las muestras fueron tomadas en condiciones de asepsia en bolsas estériles por un Médico Veterinario previamente entrenado.

Las muestras se procesaron siguiendo las metodologías descritas en el Cuadro 1. En el caso de las muestras para la detección de Salmonella se realizó una dilución 1:2 para evitar falsos negativos, y se usó como control del ensayo la cepa de Salmonella Typhimurium ATCC 14028. La prevalencia de muestras positivas para Salmonella spp se determinó mediante la siguiente ecuación: Prevalencia $=\left(\mathrm{N} .{ }^{\circ}\right.$ muestras positivas $/ \mathrm{N} .^{\circ}$ muestras totales) * 100 (Bonita et al., 2008). 


\section{Detección de Metales}

Para este caso, el muestreo se hizo a conveniencia. En 19 de las 22 granjas se tomaron muestras de concentrado para dos etapas productivas ( $\mathrm{n}=38$ muestras). Cada muestra fue analizada por triplicado.

Las muestras fueron enviadas a Tecnimicro Laboratorio de Análisis S.A.S. (Colombia) y analizadas por triplicado. Los metales analizados fueron cadmio (Cd), arsénico $(\mathrm{As})$, plomo $(\mathrm{Pb})$ y mercurio $(\mathrm{Hg})$. Para la detección de los tres primeros se empleó el método Inductively Coupled Plas$m a$ (ICP) por espectrometría de absorción atómica (EAA). Para la detección del mercurio se empleó el EPA Método 7473 (EPA, 2007). El límite de detección de la técnica fue de $<0.001 \mathrm{ppm}$.

\section{Resultados}

\section{Análisis Microbiológicos}

Se procesaron 391 muestras. De estas, 147 de Antioquia, 162 de Valle del Cauca, 42 de Cundinamarca y 42 de Meta. Todas las muestras cumplieron con los parámetros microbiológicos, recuento de Clostridium sulfito reductor, recuento de bacterias mesófilas aerobias y recuento de hongos y levaduras señalado por el Instituto Colombiano Agropecuario (ICA, 1999).

Con relación a Salmonella spp, solo una muestra obtenida en el Valle del Cauca, de la etapa de precebo, dio positiva, indicando una prevalencia de $0.25 \%$. Así mismo, cuatro muestras resultaron positivas a E. coli, dando una prevalencia de $1 \%$.

\section{Análisis de Metales}

Las dos muestras provenientes de una granja de Antioquia presentaron concentraciones por encima de la norma: $2.79 \mathrm{ppm}$ en el concentrado para la etapa de precebo y
$3.55 \mathrm{ppm}$ para la etapa de ceba. Cabe indicar que en Colombia no hay norma vigente para metales en concentrados animales; por tanto, se comparó con el Reglamento Técnico 1275/2013 de la Unión Europea (máximo 2.0 ppm) (Diario Oficial de la Unión Europea, 2013). Las muestras analizadas para cadmio y plomo cumplieron con la norma de la Unión Europea $(0.5$ ppm y 5 ppm, respetivamente). Para el caso de mercurio, todas las muestras fueron positivas para este metal, pero se encontraban dentro de los límites establecidos (en este caso se comparó con la norma chilena: 0.1 ppm; SAG, 2013).

\section{Discusión}

Los metales son considerados como sustancias no deseables en los concentrados animales, debido a su efecto en la salud humana y su capacidad de bioacumulación (Aleixieva et al., 2007; Cabella y Eguren, 2015), por lo que su presencia está sujeta a límites estrictos. En esta investigación solo se encontraron dos muestras de concentrados con presencia por encima del límite para arsénico. Los otros metales analizados no superaron los límites establecidos sugiriendo que la calidad de los concentrados que se emplean en las granjas estudiadas es adecuada.

Un estudio en Bulgaria indicó que las materias primas empleadas en los concentrados para alimentación de cerdos excedían la concentración de cadmio, donde la mayor concentración fue aportada por el aceite de girasol y la harina de pescado (Aleixieva et al., 2007). Esto mismo fue reportado por Zhang et al. (2012) en China, donde el 31.4\% de los alimentos concentrados para cerdo superaban el límite establecido por el gobierno chino $(2 \mathrm{ppm})$. Este metaloide es usado en China para mejorar la ganancia de peso y la pigmentación de los animales, práctica que obedece no solo para la mejora productiva, sino, además, por creencias culturales ( $\mathrm{Li}$ y Chen, 2005). Por otro lado, se ha establecido 
que los metales pueden ingresar a los concentrados animales a través de algunas de las materias primas que se emplean; por ejemplo, la harina de pescado puede contener altas concentraciones de mercurio, mientras que elementos no esenciales como plomo y cadmio pueden ingresar como resultado del proceso de elaboración de los concentrados y por la contaminación ambiental (Wang et al., 2013).

Para el caso del concentrado que presentó altas concentraciones de arsénico en este estudio, se evaluaron los posibles factores que podían contribuir a la presencia de este metaloide, determinándose que en la zona donde se encuentra la granja porcina no hay explotaciones mineras ni industriales, siendo una zona netamente agrícola, lo que sugiere que el arsénico ingresó por alguna de las materias primas, posiblemente por la roca fosfórica (O’Neill, 1990). Otro aspecto que pudo contribuir es el uso de aditivos como Rosarxona (3-nitro-4-hydroxylphenylarsenonic acid, ROX) y ácido arsanílico (4aminobenzenearsonic acid, ASA) (Yao et al., 2017), sustancias que están disponibles en el país.

La frecuencia de contaminación con Salmonella es baja (0.5\%), resultado similar al obtenido en Texas por Hsieh et al. (2016) quienes reportaron una prevalencia nula, mientras que Harris et al. (1997) reportó $2.8 \%$ en concentrados y $46.7 \%$ en las materias primas empleadas. Diversos autores han señalado a los concentrados como la principal fuente de introducción de Salmonella en las granjas y la cadena alimentaria (Korsak et al., 2003; EFSA, 2008; Wilkins et al., 2010). La contaminación puede ocurrir por fallas en los procesos de producción, después del mismo o al realizar la mezcla del concentrado en las granjas (Coma, 2003; Davies et al., 2004), así como por el uso de materias primas contaminadas (Wierub y Kristoffersen, 2014). En Colombia, los concentrados son usualmente suministrados por empresas que cuentan con buenas prácticas de manufactura, lo cual favorece la estandari- zación en la calidad de los concentrados; adicionalmente, la vigilancia de estas empresas, así como la importación de materias primas es realizada por el ICA, por lo que los resultados obtenidos son coherentes con estas políticas.

E. coli es un microorganismo indicador de contaminación fecal, de allí que su presencia sugiere la ocurrencia de una contaminación cruzada, pues el concentrado estaba almacenado en tolvas en varias de las granjas evaluadas, donde cabe la posibilidad de contaminación con heces de roedores o de aves, si no hay un cierre hermético. Asimismo, se ha establecido que una fuente de contaminación es el medio ambiente, donde $E$. coli puede sobrevivir hasta seis meses (Van Beers-Schreurs et al., 1992). En este estudio no se pudo analizar si las E. coli encontradas en el concentrado pertenecían a los serotipos patógenos responsables de causar cocobacilosis (Fairbrother et al., 2005), por lo que solo se les consideró como contaminantes fecales.

Por último, todas las muestras cumplieron con los límites permitidos en la legislación colombiana sobre los otros grupos indicadores analizados (recuento de bacterias mesófilas, recuento de hongos y levaduras y recuento de Clostridium sulfito reductor), sugiriendo un control adecuado en el proceso de elaboración de los concentrados, de allí que la pérdida de la calidad se daría en el manejo en granja.

\section{Conclusiones}

- El riesgo de contaminación de los cerdos por Salmonella a través del alimento balanceado en Colombia es bajo (solo una muestra resultó positiva [0.25\%]).

- Solo 2 de 38 muestras de concentrado para porcinos se encontraron por fuera del límite internacionalmente aceptable de arsénico, mientras que el plomo, cadmio y mercurio se encontraron dentro de los rangos aceptados. 


\section{Agradecimientos}

Proyecto financiado por el Ministerio de Agricultura y Desarrollo Rural, bajo el convenio N..$^{\circ} 20150360$. Los autores expresan su agradecimiento a Julián Idarraga, César Echeverry y Geraldine Hernández por su apoyo en la toma de muestras en las granjas.

\section{Literatura Citada}

1. Aleixieva D, Chobanova S, Ilchev A. 2007. Study of the level of heavy metal contamination in feed materials and compound feed for pigs and poultry in Bulgaria. Trakia J Sci 5: 61-66.

2. [APHA] American Public Health Association. 2015. Compendium of methods for the microbiological examination of foods. $5^{\text {th }}$ ed. Washington DC, USA: APHA Press. 955 p.

3. Baer A, Miller M, Dilger A. 2013. Pathogens of interest to the pork industry: a review of research on interventions to assure food safety. Compr Rev Food Sci Food Saf 12: 183-217. doi: 10.1111/15414337.12001

4. Bernard A, Broeckaert F, De Poorter $G$, De Cock A, Hermans C, Saegerman C, Houins G. 2002. The Belgian PCB/dioxin incident: analysis of the food chain contamination and health risk evaluation. Environ Res 88: 1-18. doi: 10.1006/enrs.2001.4274

5. Binter C, Straver JM, Häggblom P, Bruggeman G, Lindqvist A, Zentek J, Andersson MG 2011. Transmission and control of Salmonella in the pig feed chain: a conceptual model. Int J Food Microbiol 145: 7-17. doi: 10.1016/ j.ijfoodmicro.2010.09.001

6. Bird P, Flannery J, Crowley E, Agin JR, Goins D, Monteroso L. 2016. Evaluation of the $3 \mathrm{M}$ molecular detection assay (MDA) 2 - Salmonella for the detection of Salmonella spp in select foods and environmental surfaces: collaborative study, first action 2016.01.
J AOAC Int 99: 980-997. doi: 10.5740/ jaoacint.16-0085

7. Bonita R, Beaglehole R, Kjellström T. 2008. Epidemiología básica. $2^{\circ}$ ed. EEUU: Organización Panamericana de la Salud. 269 p.

8. Borbolla G, González J, Lopera P, Naranjo $\mathrm{H}$, Calle C. 2002. Evaluación de dos concentrados para cerdos en sus diferentes etapas de producción. Despertar Lechero 20: 103-119.

9. Boughton C, Leonard FC, Egan J, Kelly G, O'Mahony P, Markey BK, Griffin M. 2004. Prevalence and number of Salmonella in Irish retail pork sausages. J Food Prot 67: 1834-1839.

10. Cabella D, Eguren E. 2015. Buenas prácticas en la elaboración de alimentos para animales. Uruguay: Ministerio de Agricultura y Pesca. 40 p.

11. Coma J. 2003. Salmonella control in pork: effect of animal nutrition and feeding. Pig News Info 24: 49N-62N.

12. Davies J, Scott Hurd S, Funk J, Fedorka-Cray, P, Jones F. 2004. The role of contaminated feed in the epidemiology and control of Salmonella enterica in pork production. Foodborne Pathog Dis 1: 202-215. doi: 10.1089/ fpd.2004.1.202 10.1089/fpd.2004.1.202

13. den Hartog J. 2003. Feed for food: HACCP in the animal feed industry. Food Control 14: 295-99. doi: 10.1016/S09567135(02)00111-1

14. Diario Oficial de la Unión Europea. 2013. Reglamento (UE) $N^{\circ} 1275 / 2013$ de la Comisión de 6 de diciembre de 2013 por el que se modifica el anexo I de la Directiva 2002/32/CE del Parlamento Europeo y del Consejo en lo que respecta a los niveles máximos de arsénico, cadmio, plomo, nitritos, esencia volátil de mostaza e impurezas botánicas perjudiciales. L 328/86. [Internet]. Disponible en: https://www.boe.es/doue/ 2013/328/L00086-00092.pdf

15. [EFSA] European Food Safety Authority. 2008. Scientific opinion of the panel on biological hazards on a request 
from the health and consumer protection, directorate general, European commission on Microbiological risk assessment in feedingstuffs for food-producing animals. Scientific opinion of the Panel on Biological Hazards. EFSA J 720: 1-84.

16. [EPA] Environmental Protection Agency. 2007. Method 7473. Mercury in solids and solutions by termal descomposition, amalgamation, and atomic absortion spectrophotometry. [Internet]. Available in: https:// www.epa.gov/sites/production/files/ 2015-07/documents/epa-7473.pdf

17. Fairbrother JM, Nadeau E, Gyles GL. 2005. Escherichia coli in postweaning diarrhea in pigs: an update on bacterial types, pathogenesis, and prevention strategies. Anim Health Res Rev 6: 1739. doi: 10.1079/AHR2005105

18. [ICA] Instituto Colombiano Agropecuario. 1999. Directivas técnicas de alimentos para animales y sales mineralizadas. DIP-30-100-003: alimentos para animales. Parámetros microbiológicos. [Internet]. Disponible en: https:/ /www.ica.gov.co/getdoc/7d27ee5ec fe 4-47a2-868 e-7c53f4e49473/ Directivastecnicasalimentosanimales.aspx

19. [ICA] Instituto Colombiano Agropecuario. 2016. Censo porcino nacional 2016. https://www.ica.gov.co/getdoc/ 8232 c 0 e 5 - be $97-42$ bd-b 07 b $9 \mathrm{c} \mathrm{d} \mathrm{b} \mathrm{fb} 07 \mathrm{f} \mathrm{c} \mathrm{a} \mathrm{c} \mathrm{/} \mathrm{C} \mathrm{e} \mathrm{n} \mathrm{s} \mathrm{o} \mathrm{s} \mathrm{-}$ 2012.aspx\%20Consultado\%202/11/ 2017\%7D

20. Harris IT, Fedorka-Cray PJ, Gray JT, Thomas LA, Ferris K. 1997. Prevalence of Salmonella organisms in swine feed. J Am Vet Med Assoc 210: 382-385.

21. Hoogenboom L, Kan CA, Bovee T, van der Weg G, Onstenk C, Traag WA. 2004. Residues of dioxins and PCBs in fat of growing pigs and broilers fed contaminated feed. Chemosphere 57: 35 42. doi: 10.1016/j.chemos-phere.2004.04.057
22. Hsieh J, Poole T, Runyon M, Hume M, Herrman T. 2016. Prevalence of nontyphoidal Salmonella and Salmonella strains with conjugative antimicrobial-resistant serovars contaminating animal feed in Texas. J Food Prot 79: 194204. doi: 10.4315/0362-028X.JFP-15-163

23. Kant CA, Meijeir GA. 2007. The risk of contamination of food with toxic substances present in animal feed. Anim Feed Sci Technol 133: 84-108. doi: 10.1016/j.anifeedsci.2006.08.005

24. Korsak N, Jacob B, Groven B, Etienne G, China B, Ghafir Y, Daube G 2003. Salmonella contamination of pigs and pork in an integrated pig production system. J Food Prot 66: 1126-1133.

25. Li Y, Chen, T. 2005. Concentrations of additive arsenic in Beijing pig feeds and the residues in pig manure. Resour Conserv Recyc 45: 356-367. doi: 10.1016/j.resconrec.2005.03.002

26. Molla B, Sterman A, Mathews J, Artuso-Ponte V, Abley M, Farmer W, Rajala-Schultz P, et al. 2010. Salmonella enterica in commercial swine feed and subsequent isolation of phenotypically and genotypically related strains from fecal samples. Appl Environ Microbiol 76: 7188-7193. doi: 10.1128/ AEM.01169-10

27. O'Neill P. 1990. Arsenic. In: Alloway BJ (ed). Heavy metals in soils. London: Ed Blackie. p 105-119.

28. Österberg J1, Vågsholm I, Boqvist S, Lewerin SS. 2006. Feed-borne outbreak of Salmonella cubana in Swedish pig farms: risk factors and factors affecting the restriction period in infected farms. Acta Vet Scand 47:1322. doi: 10.1186/1751-0147-47-13

29. [SAG]. Ministerio de Agricultura. Gobierno de Chile. 2013. Establece límites máximos de contaminantes en insumos destinados a la alimentación animal. [Internet]. Disponible en: http:// www.sag.cl/sites/default/files/lim_max _contaminantes_insumos_oct_2013.pdf 
30. Shirota K, Katoh H, Itto T, Otsuki K. 2001. Salmonella contamination in commercial layer feed in Japan. J Vet Med Sci 62: 789-791.

31. van Beers-Schreurs HM, Vellenga L, Wensing T, Breukink HJ. 1992. The pathogenesis of the post-weaning syndrome in weaned piglets. A review. Vet Q 14: 29-34. doi: 10.1080/ 01652176.1992 .9694322

32. Wang H, Dong Y, Yang Y, Toor G, Zhang Y. 2013. Changes in heavy metal contents in animal feeds and manures in an intensive animal production region of China. J Environ Sci 25: 2435-2442. doi: 10.1016/S1001-0742(13)60473-8

33. Wierub M, Kristoffersen T. 2014. Prevention of Salmonella contamination of finished soymeal used for animal feed by a Norwegian production plant despite frequent Salmonella contamination of raw soy beans, 1994-2012. Acta Vet Scand 56: 41. doi: 10.1186/s13028-014-0041-7

34. Wilkins W, Rajic A, Waldner C, McFall M, Chow E,Muckle A, Rosengren L. 2010. Distribution of Salmonella serovars in breeding, nursery, and grow-to-finish pig, and risk factors for shedding in ten farrow-to-finish swine farms in Alberta and Saskatchewan. Can J Vet Res 74: 81-90.

35. Yao L, Huang L, He Z, Zhou CH, Li G. 2017. Occurrence of arsenic impurities in organoarsenics and animal feeds. J Agric Food Chem 61: 320-324. doi: 10.1021/jf3045022

36. Zhang F, Li X, Yang M, Li W. 2012. Content of heavy metals in animal feeds and manures from farms of different scales in northeast China. Int J Environ Res Public Health 9: 2658-2668. doi: 10.3390/ijerph9082658 